\title{
Haemophilus haemolyticus
}

National Cancer Institute

\section{Source}

National Cancer Institute. Haemophilus haemolyticus. NCI Thesaurus. Code C86430.

A species of facultatively anaerobic, Gram negative, coccobacilli shaped bacteria assigned to the phylum Proteobacteria. This species is urease, catalase and oxidase positive, porphyrin negative and requires both $\mathrm{X}$ and $\mathrm{V}$ factors to grow in culture. $\mathrm{H}$. haemolyticus is normally nonpathogenic but may cause disease in immunocompromised patients. 